\title{
Performance of cardiopulmonary resuscitation feedback systems in a long-distance train with distributed traction
}

\author{
Digna M. González-Otero ${ }^{\mathrm{a}, *}$, Sofía Ruiz de Gauna ${ }^{\mathrm{a}}$, Jesus Ruiz ${ }^{\mathrm{a}}$, Raquel Rivero ${ }^{\mathrm{a}}$, \\ J.J. Gutierrez ${ }^{\mathrm{a}}$, Purificación Saiz ${ }^{\mathrm{a}}$ and James K. Russell ${ }^{\mathrm{b}}$ \\ ${ }^{\mathrm{a}}$ Department of Communications Engineering, University of the Basque Country (UPV/EHU), Bilbao, \\ Spain \\ ${ }^{\mathrm{b}}$ Department of Emergency Medicine, Oregon Health and Science University, Portland, OR, USA
}

Received 28 February 2018

Accepted 27 March 2018

\begin{abstract}
.
BACKGROUND: Out-of-hospital cardiac arrest is common in public locations, including public transportation sites. Feedback devices are increasingly being used to improve chest-compression quality. However, their performance during public transportation has not been studied yet.

OBJECTIVE: To test two CPR feedback devices representative of the current technologies (accelerometer and electromagnetic-field) in a long-distance train.

METHODS: Volunteers applied compressions on a manikin during the train route using both feedback devices. Depth and rate measurements computed by the devices were compared to the gold-standard values.

RESULTS: Sixty-four 4-min records were acquired. The accelerometer-based device provided visual help in all experiments. Median absolute errors in depth and rate were $2.4 \mathrm{~mm}$ and 1.3 compressions per minute $(\mathrm{cpm})$ during conventional speed, and $2.5 \mathrm{~mm}$ and $1.2 \mathrm{cpm}$ during high speed. The electromagnetic-field-based device never provided CPR feedback; alert messages were shown instead. However, measurements were stored in its internal memory. Absolute errors for depth and rate were $2.6 \mathrm{~mm}$ and $0.7 \mathrm{cpm}$ during conventional speed, and $2.6 \mathrm{~mm}$ and $0.7 \mathrm{cpm}$ during high speed.

CONCLUSIONS: Both devices were accurate despite the accelerations and the electromagnetic interferences induced by the train. However, the electromagnetic-field-based device would require modifications to avoid excessive alerts impeding feed-
\end{abstract} back.

Keywords: Cardiopulmonary resuscitation (CPR), CPR feedback, CPR quality, chest compressions, public access defibrillation (PAD), train

\section{Introduction}

In case of out-of-hospital cardiac arrest, the effective application of the chain of survival, including

\footnotetext{
${ }^{*}$ Corresponding author: Digna M. González-Otero, Department of Communications Engineering, University of the Basque Country (UPV/EHU), Torres Quevedo Plaza, n1, 48013 Bilbao, Spain. Tel.: +34 946018255; Fax: +34 946014259; E-mail: dignamaria.gonzalez@ehu.eus.
}

0928-7329/18/\$35.00 (c) 2018 - IOS Press and the authors. All rights reserved

This article is published online with Open Access and distributed under the terms of the Creative Commons Attribution NonCommercial License (CC BY-NC 4.0). 
the immediate initiation of cardiopulmonary resuscitation (CPR), is pivotal to maximize the chances of survival of the patient [1-4]. To maximize survival rates, chest compressions should be administered with a depth of between 5 and $6 \mathrm{~cm}$, at a rate of between 100 and 120 compressions per minute, allowing full chest release between compressions and minimizing interruptions [5]. However, poor quality chest compressions are frequently provided both by laypeople [6] and by well-trained rescuers $[7,8]$.

Defibrillator manufacturers have developed CPR aid devices that monitor chest compressions in real time and provide feedback to the rescuers [9-12]. These systems can be standalone devices or work connected to defibrillators. Although a beneficial effect on patient outcomes has yet to be established [13], there is good evidence that the use of real-time feedback devices increases adherence to CPR quality guidelines [5,13,14].

Recent deployment of public access defibrillation (PAD) programs intends to provide CPR training and defibrillation availability to the general public. PAD locations include means of transport such as airplanes, cruise ships and, more recently, intercity trains [15-18]. In the particular case of moving trains, we hypothesized that CPR feedback devices could be affected by severe environmental electromagnetic interferences and by the movement during the journey.

The aim of this study was to assess the reliability and accuracy of two devices representative of the main existing technologies in a moving long-distance train.

\section{Materials and methods}

\subsection{Study protocol}

We used two standalone feedback devices representative of the two existing technologies: an accelerometer-based feedback device (System A), and an electromagnetic-field-based device (System B).

The aim of the study was to evaluate the reliability and accuracy of both feedback devices to monitor and guide chest compression rate and depth in a simulated cardiac arrest scenario in a long-distance train. We used a Resusci Anne CPR manikin (Laerdal Medical, Norway) equipped with a linear resistive sensor (SP1-4, Celesco, USA) to measure the reference chest displacement signal, as shown in Fig. 1. Four volunteers (laypeople) were recruited in our university. They received basic CPR training and were instructed in the use of CPR feedback systems. The ethical committee for research involving human subjects of the University of the Basque Country (CEISH UPV/EHU BOPV 32, 17-2-2014) approved the experimental protocol (M10-2015-208-RUIZ-OJEDA).

Measurements were performed in the four-car Alvia S-120 train operated by Renfe railway company (Spain). This train has distributed traction. The unit comprises eight asynchronous motors, two per car, and it has two driver's cabs, where the control and driving equipment is installed. The units can be powered at $3000 \mathrm{~V} \mathrm{DC}$ (conventional speed) or $25000 \mathrm{~V}$ with $50 \mathrm{~Hz} \mathrm{AC} \mathrm{(high} \mathrm{speed).} \mathrm{The} \mathrm{experiments}$ were performed during the Bilbao-Barcelona-Bilbao route (approximately $1320 \mathrm{~km}$ for the round-trip journey), which comprises both a conventional and a high-speed interval. Mean velocity was $86 \mathrm{~km} / \mathrm{h}$ and $166 \mathrm{~km} / \mathrm{h}$ for the conventional and for the high-speed intervals, respectively. The manikin was placed over the synthetic carpeting that covered the floor of the car.

Rescuers were randomly grouped in couples. Each couple provided CPR to the manikin for 4 min using either System A or System B, alternating series of 30 chest compressions with 5-s pauses, and changing rescuers after 2 min. This protocol was repeated for the two couples, for both feedback devices and for two target depths: 50 and $35 \mathrm{~mm}$, to cover a wide range of depths expected to be found 


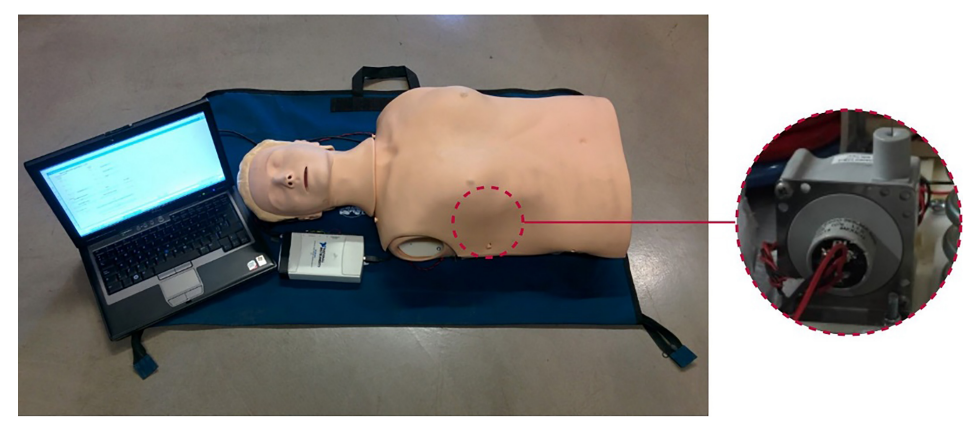

Fig. 1. Experimental setup. Manikin fitted with a displacement sensor, acquisition card and laptop computer.

in real cardiac arrest events. Target rate was $100 \mathrm{cpm}$ for all the measurements, and was guided by a metronome. Measurements were repeated at different points of the route, and the order of the experiments was randomized. A total of 404 -min records were acquired per device during the conventional interval and 24 during the high-speed interval.

\subsection{Data analysis and performance evaluation}

The reliability of both systems was analyzed by evaluating the number of episodes for which they provided feedback to the rescuer during the whole record. Additionally, the accuracy of the feedback devices was evaluated by measuring the errors in depth and rate estimation. Errors were computed as the difference between the values stored by each device and the values obtained from the reference compression depth signal acquired by the resistive sensor. Kruskal-Wallis test was used to perform between-groups comparisons, and $p$-values $<0.05$ were considered significant.

\section{Results}

\subsection{Feedback reliability}

System A provided visual feedback for all the acquired episodes. In contrast, System B failed to provide visual feedback in all the episodes. In most cases (77\%) it showed an alert indicating that the back pad was at an incorrect distance from the chest pad, and even if we tried to adjust the position of the pads, the alert persisted. In the rest of the cases (23\%) it showed another alert indicating that electronic noise was interfering with the device.

\subsection{Accuracy on measurement of depth and rate}

Despite System B failed to provide visual help, both devices internally stored and computed depth and rate values for each chest compression. Figure 2 shows boxplots of the error in the measurement of compression depth (top panel) and rate (bottom panel) for both devices, during conventional and high-speed intervals. Statistically significant differences were found between errors in depth estimation of both devices, both globally and when each scenario (conventional and high-speed) was considered separately ( $p<0.001$ in all cases). Distribution of error in depth estimation was also different between scenarios, both globally and for each device $(p<0.001)$. Regarding rate calculation, statistically significant differences were found between devices, although with a smaller significance level than for depth 

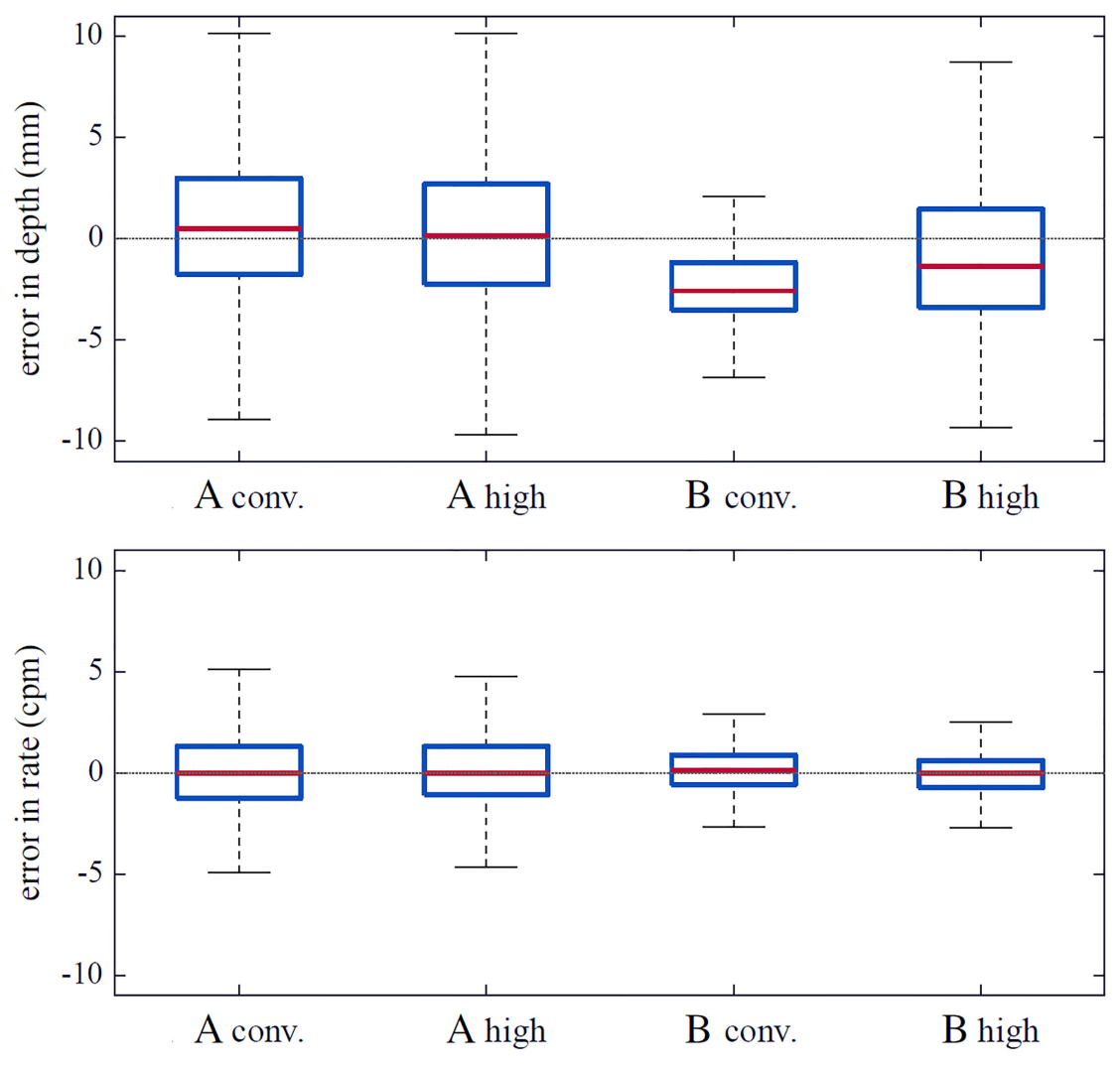

Device and route interval

Fig. 2. Distribution of the error in depth and rate calculation for both feedback devices, System A and System B, during the conventional and the high-speed intervals of the train route.

Table 1

Unsigned error in depth and rate calculation for both devices in a long-distance train, expressed as median (Mdn), inter-quartile range $(\mathrm{IQR})$ and 95 th percentile $\left(P_{95}\right)$

\begin{tabular}{|c|c|c|c|c|}
\hline \multirow[b]{2}{*}{ Device } & \multicolumn{2}{|c|}{ Depth (mm) } & \multicolumn{2}{|c|}{ Rate (cpm) } \\
\hline & Mdn (IQR) & $P_{95}$ & Mdn (IQR) & $P_{95}$ \\
\hline \multicolumn{5}{|l|}{ A } \\
\hline Conventional & $2.4(1.1-4.5)$ & 10.2 & $1.3(0.7-2.0)$ & 3.2 \\
\hline High speed & $2.5(1.1-4.8)$ & 10.9 & $1.2(0.6-2.0)$ & 3.4 \\
\hline \multicolumn{5}{|l|}{ B } \\
\hline Conventional & $2.6(1.6-3.5)$ & 5.4 & $0.7(0.4-1.3)$ & 2.2 \\
\hline High speed & $2.6(1.4-4.3)$ & 8.0 & $0.7(0.3-1.2)$ & 2.3 \\
\hline
\end{tabular}

calculations $(p=0.019)$. Finally, each device showed weak but statistically significant differences in rate between scenarios $(p=0.047)$.

Table 1 shows the median (IQR) and the 95th percentile of the unsigned (absolute) error in depth and rate estimation for each feedback system in both scenarios. Median error in depth estimation was between 2.4 and $2.6 \mathrm{~mm}$ depending on the device and on the scenario, with a 95th percentile below $11 \mathrm{~mm}$. Median error in rate estimation was between 0.7 and $1.3 \mathrm{cpm}$, with a 95th percentile below 3.5 cpm in all cases. 


\section{Discussion}

Public access defibrillation programs that place AEDs in public settings and train laypersons in CPR and AED usage have demonstrated survival benefit [5]. AEDs are being used by trained laypersons in casinos [19], airports [20], bus and railway stations [21], shopping malls, sports facilities, and schools and universities. AEDs continue to be increasingly deployed in locations with high risk for cardiac arrests (usually high-density public areas) or remote from any chance of conventional treatment, such as commercial aviation [15,16,22], ships [17,23], or long-distance trains [18]. Several studies identified train stations as the public location with the highest incidence of cardiac arrests [24-27]. Unfortunately, it is difficult to ascertain the precise incidence of cardiac arrests occurring on board moving trains; reports of these events on the scientific literature are sparse, and in most of them cardiac arrests occurring on board trains are usually reported together with those occurring in train stations. Only one study performed in Tokyo and its surroundings separately reported that $12 \%$ of the total arrests occurring in trains happened on board train vehicles [18]. Deploying CPR feedback devices together with AEDs on board long-distance trains would further support the treatment of cardiac arrest victims in this scenario, as it would help to improve CPR quality, which has proved to be key for patient survival.

To our knowledge, there is no other study assessing the performance of CPR feedback devices in moving trains. We identified only two studies which evaluated the susceptibility of AEDs in train stations $[28,29]$. In our experiments, environmental conditions in the train severely affected the electromagnetic-field based device, as it failed to provide feedback to the rescuer. However, the device did store CPR performance information in all cases, and both depth and rate were accurately measured. On the other hand, the acceleration-based device did fulfill its function in the train.

Both devices had potential to be used in a moving train, although the electromagnetic-field basic device would require modifications to avoid excessive alerts impeding visual help. The deployment of CPR feedback devices together with AEDs when implementing PAD programs in trains could improve CPR quality and contribute to a better implementation of the chain of survival.

However, care should be taken when extrapolating the results of this study. Experiments in railways based on different technologies, such as magnetic levitation, or with very different physical characteristics, for example generating higher vibrations, could yield different results. Additionally, other feedback devices could be more sensitive to this environment. Consequently, specific studies are warranted to ensure proper performance before deploying CPR feedback devices on a certain train route.

\section{Conclusions}

Environmental conditions in a moving train did not affect the ability of the assessed CPR feedback devices to accurately compute and store measurements of compression depth and rate during CPR. However, the electromagnetic-field-based device might require modifications to avoid excessive alerts impeding visual help. Availability of CPR aid in addition to AED deployment aboard long-distance trains could contribute to the effective treatment of cardiac arrest victims.

\section{Acknowledgments}

This manuscript derives from the thesis work "Feedback systems for the quality of chest compressions during cardiopulmonary resuscitation" [30] by coauthor Digna M. González-Otero, under the supervision of coauthors Jesus Ruiz and Sofia Ruiz de Gauna. 
This work received financial support from the Spanish Government through the project TEC201231144, from the Basque Government through the grant BFI-2011-166, and through the Vice Chancellor's office for Research of the University of the Basque Country (UPV/EHU) through the grant "Contratación de doctores recientes hasta su integración en programas de formación postdoctoral".

The authors would like to thank all the volunteers who participated in the data collection. Thanks to Renfe for its support in the performance of the experiments.

\section{Conflict of interest}

The authors declare that there is no conflict of interest regarding the publication of this paper.

\section{References}

[1] Nolan J, Soar J, Eikeland H. The chain of survival. Resuscitation. 2006; 71(3): 270-271. https://www.ncbi.nlm.nih.gov/ pubmed/29710761.

[2] Sasson C, Rogers MA, Dahl J, Kellermann AL. Predictors of survival from out-of-hospital cardiac arrest. Circulation: Cardiovascular Quality and Outcomes. 2010; 3(1): 63-81. https://content.iospress.com/articles/technology-and-healthcare/thc181241.

[3] Hollenberg J, Herlitz J, Lindqvist J, Riva G, Bohm K, Rosenqvist M, et al. Improved survival after out-of-hospital cardiac arrest is associated with an increase in proportion of emergency crew-witnessed cases and bystander cardiopulmonary resuscitation. Circulation. 2008; 118(4): 389-396.

[4] Wissenberg M, Lippert FK, Folke F, Weeke P, Hansen CM, Christensen EF, et al. Association of national initiatives to improve cardiac arrest management with rates of bystander intervention and patient survival after out-of-hospital cardiac arrest. Jama. 2013; 310(13): 1377-1384.

[5] Perkins GD, Handley AJ, Koster RW, Castrén M, Smyth MA, Olasveengen T, et al. European Resuscitation Council Guidelines for Resuscitation 2015. Resuscitation. 2015; 95: 81-99.

[6] Gallagher EJ, Lombardi G, Gennis P. Effectiveness of bystander cardiopulmonary resuscitation and survival following out-of-hospital cardiac arrest. JAMA: the Journal of the American Medical Association. 1995; 274(24): 1922-1925.

[7] Abella BS, Alvarado JP, Myklebust H, Edelson DP, Barry A, O'Hearn N, et al. Quality of cardiopulmonary resuscitation during in-hospital cardiac arrest. JAMA: the Journal of the American Medical Association. 2005 Jan; 293(3): 305-310.

[8] Wik L, Kramer-Johansen J, Myklebust H, Sørebø H, Svensson L, Fellows B, et al. Quality of cardiopulmonary resuscitation during out-of-hospital cardiac arrest. JAMA: the Journal of the American Medical Association. 2005 Jan; 293(3): 299-304.

[9] Yeung J, Meeks R, Edelson D, Gao F, Soar J, Perkins GD. The use of CPR feedback/prompt devices during training and CPR performance: a systematic review. Resuscitation. 2009; 80(7): 743-751.

[10] Krasteva V, Jekova I, Didon JP. An audiovisual feedback device for compression depth, rate and complete chest recoil can improve the CPR performance of lay persons during self-training on a manikin. Physiological Measurement. 2011; 32(6): 687.

[11] Pozner CN, Almozlino A, Elmer J, Poole S, McNamara D, Barash D. Cardiopulmonary resuscitation feedback improves the quality of chest compression provided by hospital health care professionals. The American Journal of Emergency Medicine. 2011; 29(6): 618-625.

[12] Gruber J, Stumpf D, Zapletal B, Neuhold S, Fischer H. Real-time feedback systems in CPR. Trends in Anaesthesia and Critical Care. 2012; 2(6): 287-294.

[13] Kirkbright S, Finn J, Tohira H, Bremner A, Jacobs I, Celenza A. Audiovisual feedback device use by health care professionals during CPR: a systematic review and meta-analysis of randomised and non-randomised trials. Resuscitation. 2014; 85(4): 460-471.

[14] AHA MFH, Nolan JP. 2015 International Consensus on Cardiopulmonary Resuscitation and Emergency Cardiovascular Care Science With Treatment Recommendations. Circulation. 2015; 132: S1.

[15] Goodwin T. In-flight medical emergencies: an overview. BMJ: British Medical Journal. 2000; 321(7272): 1338.

[16] Page RL, Joglar JA, Kowal RC, Zagrodzky JD, Nelson LL, Ramaswamy K, et al. Use of automated external defibrillators by a US airline. New England Journal of Medicine. 2000; 343(17): 1210-1216.

[17] Oldenburg M, Baur X, Schlaich C. Implementation of automated external defibrillators on merchant ships. Journal of Travel Medicine. 2011; 18(4): 233-238. 
[18] Fukuike S, Otomo Y, et al. Cardiac arrest cases and automated external defibrillator use in railroad stations in Tokyo. International Journal of Clinical Medicine. 2014; 5(20): 1328.

[19] Valenzuela TD, Roe DJ, Nichol G, Clark LL, Spaite DW, Hardman RG. Outcomes of rapid defibrillation by security officers after cardiac arrest in casinos. The New England Journal of Medicine. 2000 Oct; 343(17): 1206-1209.

[20] Caffrey S. Feasibility of public access to defibrillation. Current Opinion in Critical Care. 2002; 8(3): 195-198.

[21] Caffrey SL, Willoughby PJ, Pepe PE, Becker LB. Public use of automated external defibrillators. New England Journal of Medicine. 2002; 347(16): 1242-1247.

[22] O'Rourke MF, Donaldson E, Geddes JS. An airline cardiac arrest program. Circulation. 1997 Nov; 96(9): $2849-2853$.

[23] Dahl E. Implementation of automated external defibrillators on German merchant ships. Journal of Travel Medicine. 2011; 18(4): 229-230.

[24] Marijon E, Bougouin W, Tafflet M, Karam N, Jost D, Lamhaut L, et al. Population movement and sudden cardiac arrest location. Circulation. 2015; 131: 1546-1554.

[25] Murakami Y, Iwami T, Kitamura T, Nishiyama C, Nishiuchi T, Hayashi Y, et al. Outcomes of out-of-hospital cardiac arrest by public location in the public-access defibrillation era. Journal of the American Heart Association. 2014; 3(2): e000533.

[26] Folke F, Lippert FK, Nielsen SL, Gislason GH, Hansen ML, Schramm TK, et al. Location of cardiac arrest in a city center: strategic placement of automated external defibrillators in public locations. Circulation. 2009; 120(6): 510-517.

[27] Muraoka H, Ohishi Y, Hazui H, Negoro N, Murai M, Kawakami M, et al. Location of Out-of-Hospital Cardiac Arrests in Takatsuki City. Circulation Journal. 2006; 70(7): 827-831.

[28] Kanz KG, Kay MV, Biberthaler P, Russ W, Wessel S, Lackner CK, et al. Susceptibility of automated external defibrillators to train overhead lines and metro third rails. Resuscitation. 2004; 62(2): 189-198.

[29] Fleischhackl R, Singer F, Roessler B, Arrich J, Fleischhackl S, Losert H, et al. Automated external defibrillators do not recommend false positive shocks under the influence of electromagnetic fields present at public locations. Anesthesia \& Analgesia. 2006; 103(6): 1485-1488.

[30] González-Otero DM. Feedback systems for the quality of chest compressions during cardiopulmonary resuscitation; 2015. 